\title{
Utilization of Laboratory Tests of a Gingival Crevicular Sample in an Occupational Periodontal Care System: A Pilot Study
}

\author{
Takashi Hanioka ${ }^{1}$, Mikako Kishimoto', Keiko Takaya ${ }^{1}$, Muneo TanakA ${ }^{1}$, \\ Yukiko Matsumori $^{1}$, Ryoichi Matsuse ${ }^{2}$, Kiyoharu Iwata ${ }^{3}$ and Satoshi Shizukuishi ${ }^{1}$ \\ 'Department of Preventive Dentistry, Osaka University Faculty of Dentistry, \\ ${ }^{2}$ Kyoto Medical Science Laboratory Inc, \\ ${ }^{3}$ The Association for Preventive Medicine of Japan
}

\begin{abstract}
Utilization of Laboratory Tests of a Gingival Crevicular Sample in an Occupational Periodontal Care System: A Pilot Study: Takashi HANIOKA, et al. Department of Preventive Dentistry, Osaka University Faculty of Dentistry-Periodontal disease leads to tooth loss in workers during their later life. Workers tend not only to be unaware of the disease but also pay transient attention to their periodontal health. Therefore a variety of information on periodontal health is requested for an occupational health promotion systems, in addition to the conventional screening approaches for periodontal disease. Subgingival plaque and gingival crevicular fluid include biological materials that pose a microbial challenge and affect the host response. The aims of this pilot study were to examine whether the gingival crevicular samples obtained from workers could be tested in bulk at a laboratory, and whether the biological markers in the samples could provide information in addition to a conventional screening approach. A total of 182 periodontal sites were assessed in 96 workers by means of the Community Periodontal Index and 10 laboratory tests. Significant relationships were found between the periodontal variables and the parameters of the laboratory test. Principal component analysis revealed four components of the laboratory tests apart from conventional periodontal variables. Explanations given for the components were serum exudate and bleeding, neutrophil, periodontal pathogen and tissue destruction. These findings indicated that laboratory tests of gingival crevicular samples offer information in addition to that obtained by means of the conventional screening approach.
\end{abstract}

(J Occup Health 2000; 42: 8-13)

Received Jun l, 1999; Accepted Jul 28, 1999

Correspondence to: T. Hanioka, Department of Preventive Dentistry, Osaka University Faculty of Dentistry, Yamadaoka 1-8, Suita, Osaka 565-0871, Japan
Key words: Laboratory test, Gingival crevicular fluid, Subgingival plaque, Occupational oral health, Periodontal disease

Occupational health in Japan is facing new problems associated with the aging of the working population and the increasing numbers of workers with stress-associated and/or lifestyle-related diseases. To address such problems, the Ministry of Labor has enforced several new more comprehensive programs. Among these, Total Health Promotion plan was implemented in 1988. The program includes oral health promotion at the workplace, to enhance periodontal care.

Periodontal tissue, which is composed of gingiva, periodontal ligament, cementum and alveolar bone, supports the dentition. Destruction of periodontal tissue, especially loss of periodontal attachment and alveolar bone, leads to tooth loss. A series of tooth loss events may result in serious disability in fundamental oral functions such as mastication and pronunciation. Periodontal disease is called 'silent disease', since subjective symptoms are mild during development of the disease until tooth loss is evident. Furthermore, a recent report indicates periodontal disease as a risk factor in systemic diseases including bacteremia, infective endocarditis, cardiovascular disease and atherosclerosis, prosthetic device infection, diabetes mellitus, respiratory diseases, and adverse pregnancy outcomes ${ }^{11}$. Therefore, early detection of the disease is important to prevent not only loss of oral functions but also life-threatening systemic diseases.

Recently, the WHO Community Periodontal Index $(\mathrm{CPI})^{2 j}$ was introduced in the field of occupational oral health care. This method is concise and easy to implement to identify periodontal disease.

Periodontal health promotion is also important in the workplace, because periodontal destruction may occur 
due to multifactorial reasons including microbial challenge and host response. Knowledge of the microbial challenge and the host response in their own periodontal tissue may be useful information for workers. Analysis of the constituents of subgingival plaque and gingival crevicular fluid (GCF) may yield information on the microbial challenge and host response.

A diagnostic test for peptidase of periodontopathic bacteria (PPB) which is produced by Treponema denticola, Porphyromonas gingivalis and Bacteroides forsythus is available ${ }^{3.41}$. GCF includes molecules which are derived from serum and locally produced factors of host origin ${ }^{5-71}$. Acute inflammatory response of the periodontal tissue to the microbial challenge has often been studied with cellular and serum-associated mediators: Transferrin (TF), $\alpha_{1}$-antitrypsin (AT) and albumin (ALB) are serum-derived proteins found in the gingival exude. Lysozyme (LYS) is an extracellular lysosomal enzyme which could identify the presence of neutrophils. Myeloperoxidase (MPO), neutrophil elastase (NE) and lactoferrin (LF) derive from different effector systems of the acute inflammatory response ${ }^{6}$. Breakdown products of connective tissue such as fibronectin (FN) could be markers of tissue destruction. Correlation between the GCF constituents and some aspects of periodontal disease has been well demonstrated and a small number of possible predictors for future periodontal destruction have been reported ${ }^{7}$.

Biochemical analyses of blood and urine are widespread in the occupational health care system in Japan. Many facilities are now capable of such analyses, and these resources could also be made available for the analysis of gingival crevicular samples in an occupational oral health care system. The gingival crevicular sample is easy to collect and may offer important information on periodontal health. The aims of this pilot study were to examine whether the crevicular samples obtained from workers could be tested in bulk at a laboratory, and whether the biological markers in the samples could provide information in addition to that provided by conventional screening methods.

\section{Subjects and Methods}

The participants in this study were 72 male and 24 female employees aged 20-58 working for a company in Japan. All participants were office personnel. Permission for the study was obtained from the company's management. Gingival crevicular samples were collected during the periodic general and oral health check-ups. Informed consent was obtained prior to data collection. The oral examinations and sample collections were performed in the company's conference room with a portable dental chair and adequate light source. Three dentists experienced in performing the examinations were advised before performing the examinations on the subjects of the study. Periodontal health was assessed with the CPI system ${ }^{21}$ and the conventional classification of periodontal disease (STG): healthy, gingivitis, and mild, moderate and advanced periodontitis.

Following the assessment of periodontal health, gingival crevicular samples were collected at two sites per subject: the mesio-palatal crevice of the right maxillary first molar and the site with the highest CPI score were selected for the sample collection. After supragingival plaque in the selected sites was removed with a cotton ball, three sterile paper points (Johnson and Johnson, Tokyo, Japan) were placed into the crevice and were left for $30 \mathrm{~s}$ to collect GCF and subgingival plaque. The paper points were stored at $-20^{\circ}$ in a sampling tube and then delivered to a laboratory for the assay of proteins and enzymes.

Elution of the constituents from the paper points was performed by gentle agitation of the samples in $400 \mu \mathrm{l}$ of $0.02 \mathrm{M}$ Tris- $\mathrm{HCl}$ buffer $(\mathrm{pH} 7.5)$ for $30 \mathrm{~min}$ at the laboratory. The samples were diluted and then assayed for the total amount of protein, for hemoglobin (HB), ALB, TF, AT, MPO, LYS, FN, NE and LF, and total activity of enzymes for PPB during $30 \mathrm{~s}$ of sample collection. The assay was performed for $\mathrm{HB}, \mathrm{ALB}, \mathrm{TF}$, AT, MPO, LYS, FN, NE and LF by sandwich enzyme linked immunosorbant assays (ELISA) ${ }^{8.91}$. Anti-human polyclonal antibodies of the HB, ALB, TF, MPO, AT and LYS (Dakopatts, Glostrup, Denmark), and FN and NE (Serotec, Oxford, England) were used in the wells of 96well microplates. Assay of the LF was completed with a commercially available kit (Shionogi \& Co., Ltd., Osaka, Japan). These analyses were performed according to the manufacturer's recommended protocols. PPB was determined with reagents in a commercially available kit (Periocheck, Sunstar Inc., Osaka, Japan) ${ }^{4}$.

Three periodontal health parameters were used for the site-based analyses; an individual code of the CPI system (CPI-I) $)^{1}$, the CPI code at the sampling site (CPI-S) and the five categories of the STG which were coded 1 to 5 for statistical analyses. The Spearman signed rank test was employed to assess relationships between two variables. Ten parameters for laboratory testing, age and the periodontal variables were entered into factor analysis for the principal component with varimax rotation using a correlation matrix ${ }^{10)}$. Common factors were analyzed in each significant principal component. These analyses were performed with the statistical package of social sciences (SPSS, SPSS Inc, Chicago, IL). Statistical significance was set at the $5 \%$ level.

\section{Results}

A total of 182 samples were obtained from 96 subjects. Only one sample was obtained from 10 subjects because the sampling site in the maxillary first molar prove to be the site with the highest CPI score for those subjects. 
Table 1 shows the distribution of the CPI score for the sampling site. About $70 \%$ of the sites had a pocket depth of less than $3 \mathrm{~mm}$ and $25 \%$ had pockets 4 or $5 \mathrm{~mm}$ deep. Pockets more than $6 \mathrm{~mm}$ deep were found at only at $1 \mathrm{l}$ sites $(6 \%)$,

Distributions of the variables of the laboratory tests

Table 1. Distribution of CPI scores at the sampling site

\begin{tabular}{|c|c|c|c|}
\hline Score & Description & $\mathrm{n}$ & $\%$ \\
\hline 0 & Healthy & 26 & 14.3 \\
\hline 1 & Bleeding & 30 & 16.5 \\
\hline 2 & Calculus & 69 & 37.9 \\
\hline 3 & Pocket 4 or $5 \mathrm{~mm}$ deep & 46 & 25.3 \\
\hline \multirow[t]{2}{*}{4} & Pocket more than $6 \mathrm{~mm}$ deep & 11 & 6.0 \\
\hline & Total & 182 & 100 \\
\hline
\end{tabular}

by the CPI score for sampling sites (CPI-S) are shown as box-and-whisker plots in Fig. 1. Wider ranges of distribution were found in sites with higher CPI-S scores. Distribution of most variables tended to be skewed to lower values. Sites with deep pockets $(\mathrm{CPJ}-\mathrm{S}=4)$ had the highest values in the 50th percentile of most variables, but distributions in sites with adjacent scores overlapped for most variables.

Correlation coefficients between variables are shown in Table 2. Conventionally used periodontal variables (STG, CPI-I and CPI-S) closely correlated with each other $(r=0.663-0.745)$. Variables in laboratory tests also correlated significantly with each other except for PPB $(r=0.271-0.947)$. Significant correlations were found between the periodontal variables and most of the laboratory tests, but correlation factors were low (less then 0.4). The CPI-S score showed significant correlation with all laboratory testing parameters.
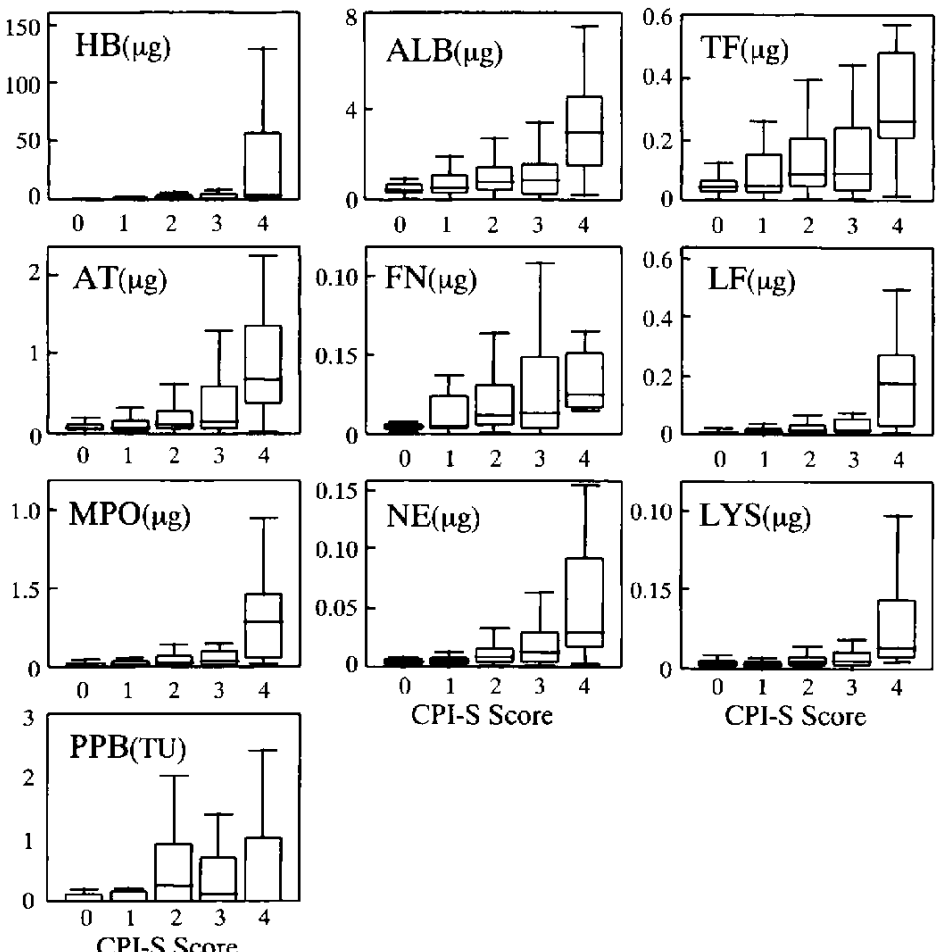

C.PI-S Score

Fig. 1. Distribution of variables from the laboratory test by using the CPI score for the sampling site. In these box plots, the top, bottom and line through the middle of the box correspond to the 75th, 25th and 50 th percentiles, respectively. The whiskers on the bottom and top extend from the smallest to the largest observed values that were not outside values (exceeding 1.5 box-lengths from the 25 th or 75 th percentile). The units are the total amount of proteins and total activity of the enzyme during 30 s of sample collection. HB: hemoglobin, ALB: albumin, TF: transferrin, AT: $\alpha_{1}$-antitrypsin, FN: fibronectin, LF: lactoferrin, MPO: myeloperoxidase, NE: neutrophil elastase, LYS: lysozyme, PPB: peptidase of periodontopathic bacteria, TU: trypsin units. 
Factor analysis was performed after confirmation of the size of communality and loadings. The first 6 principal components with varimax rotation are shown in Table 3. In the principal component analysis, variances of the first 5 components exceeded one, indicating that the components each had a significant influence on the total variance obtained from 14 variables. The components explained $83 \%$ of the total variance. In each component, factor loading with an absolute value of more than 0.6 reflects the significance of the component. Factors which significantly reflected component $I$ were $\mathrm{TF}, \mathrm{ALB}, \mathrm{NE}, \mathrm{AT}$ and $\mathrm{HB}$, in that order from highest to

Table 2. Correlation coefficients and significant levels in simple correlation analyses

\begin{tabular}{lcccccccccccccc}
\hline & Age & STG & CPI-I & CPI-S & HB & ALB & TF & AT & NE & LF & MPO & LYS & FN & PPB \\
\hline Age & - & 0.442 & 0.331 & 0.330 & 0.140 & 0.057 & 0.060 & 0.174 & 0.126 & 0.228 & 0.233 & 0.076 & 0.136 & 0.172 \\
STG & $*$ & - & 0.722 & 0.663 & 0.331 & 0.272 & 0.229 & 0.374 & 0.323 & 0.304 & 0.302 & 0.120 & 0.278 & 0.128 \\
CPI-I & $*$ & $*$ & - & 0.745 & 0.236 & 0.255 & 0.230 & 0.356 & 0.312 & 0.311 & 0.308 & 0.231 & 0.243 & 0.094 \\
CPI-S & $*$ & $*$ & $*$ & - & 0.331 & 0.316 & 0.316 & 0.338 & 0.400 & 0.372 & 0.332 & 0.326 & 0.309 & 0.190 \\
HB & NS & $*$ & $*$ & $*$ & - & 0.793 & 0.774 & 0.728 & 0.718 & 0.456 & 0.476 & 0.278 & 0.736 & 0.111 \\
ALB & NS & $*$ & $*$ & $*$ & $*$ & - & 0.947 & 0.883 & 0.863 & 0.567 & 0.546 & 0.336 & 0.874 & 0.042 \\
TF & NS & $*$ & $*$ & $*$ & $*$ & $*$ & - & 0.879 & 0.876 & 0.603 & 0.571 & 0.349 & 0.865 & 0.070 \\
AT & $*$ & $*$ & $*$ & $*$ & $*$ & $*$ & $*$ & - & 0.869 & 0.674 & 0.676 & 0.352 & 0.819 & 0.085 \\
NE & NS & $*$ & $*$ & $*$ & $*$ & $*$ & $*$ & $*$ & - & 0.760 & 0.750 & 0.452 & 0.825 & 0.134 \\
LF & $*$ & $*$ & $*$ & $*$ & $*$ & $*$ & $*$ & $*$ & $*$ & - & 0.927 & 0.685 & 0.521 & 0.117 \\
MPO & $*$ & $*$ & $*$ & $*$ & $*$ & $*$ & $*$ & $*$ & $*$ & $*$ & - & 0.654 & 0.482 & 0.166 \\
LYS & NS & NS & $*$ & $*$ & $*$ & $*$ & $*$ & $*$ & $*$ & $*$ & $*$ & - & 0.271 & 0.121 \\
FN & NS & $*$ & $*$ & $*$ & $*$ & $*$ & $*$ & $*$ & $*$ & $*$ & $*$ & $*$ & - & 0.060 \\
PPB & $*$ & NS & NS & $*$ & NS & NS & NS & NS & NS & NS & $*$ & NS & NS & - \\
\hline
\end{tabular}

STG: Stage of periodontal health, CPI-I: individual score in the CPITN system, CPI-S: CPI score for the sampling site, NE: neutrophil elastase, HB: hemoglobin, ALB: albumin, TF: transferrin, AT: $\alpha_{1}$-antitrypsin, LF: lactoferrin, MPO: myeloperoxidase, LYS: lysozyme, FN: fibronectin, PPB: peptidase of periodontopathic bacteria. *Significant at $\mathrm{p}<0.05$. NS: Not significant. $\mathrm{N}=182$ sites.

Table 3. First 6 principal components with a varimax rotation estimated from 14 variables. Factor loadings, the variance of each component and its relative and cumulative part of total variance are given

\begin{tabular}{|c|c|c|c|c|c|c|}
\hline \multirow{2}{*}{ Variables } & \multicolumn{6}{|c|}{ Component } \\
\hline & 1 & 2 & 3 & 4 & 5 & 6 \\
\hline Age & -0.002 & 0.138 & 0.247 & 0.953 & 0.047 & 0.030 \\
\hline Stage of periodontal health & 0.101 & 0.092 & 0.897 & 0.223 & 0.067 & 0.073 \\
\hline CPI score for subject & 0.129 & 0.145 & 0.897 & 0.060 & -0.014 & 0.018 \\
\hline CPI score for the sampling site & 0.147 & 0.209 & 0.730 & 0.109 & 0.056 & 0.114 \\
\hline Hemoglobin & 0.681 & 0.385 & 0.124 & 0.039 & -0.032 & 0.072 \\
\hline Albumin & 0.934 & 0.168 & 0.096 & -0.011 & -0.012 & 0.227 \\
\hline Transferrin & 0.939 & 0.196 & 0.077 & -0.006 & -0.033 & 0.090 \\
\hline$\alpha_{1}$-Antitrypsin & 0.904 & 0.331 & 0.135 & 0.051 & 0.014 & 0.148 \\
\hline Neutrophil elastase & 0.917 & 0.264 & 0.104 & 0.005 & 0.026 & 0.112 \\
\hline Lactoferrin & 0.433 & 0.837 & 0.170 & 0.143 & 0.032 & 0.012 \\
\hline Myeloperoxidase & 0.370 & 0.856 & 0.156 & 0.151 & -0.008 & -0.023 \\
\hline Lysozyme & 0.156 & 0.910 & 0.093 & -0.024 & 0.017 & 0.060 \\
\hline Fibronectin & 0.480 & 0.024 & 0.123 & 0.040 & 0.055 & 0.862 \\
\hline Peptidase of periodontopathic bacteria & -0.014 & 0.020 & 0.048 & 0.042 & 0.996 & 0.037 \\
\hline Variance & 4.504 & 2.750 & 2.343 & 1.026 & 1.010 & 0.867 \\
\hline Proportion & 0.322 & 0.196 & 0.167 & 0.073 & 0.072 & 0.062 \\
\hline Cumulative & $32.2 \%$ & $51.8 \%$ & $68.6 \%$ & $75.9 \%$ & $83.1 \%$ & $89.3 \%$ \\
\hline
\end{tabular}


lowest. All were laboratory tests. Significant variables for component 2 were also laboratory tests: LYS, MPO and LF. Periodontal variables such as the CPI-I, STG and CPI-S were significant in the third component. Age and $P P B$ were the only significant variables in components 4 and 5 , respectively. FN was significant in component 6 , but the variance of the component was less than one.

\section{Discussion}

The first aim of this study was to clarify whether the gingival crevicular samples obtained from workers could be tested in bulk at a laboratory. Significant relationships between CPI-S and all biological markers may indicate the validity of the test. Periodontal sites with severe destruction (CPI-S $=4$ ) had the highest value for each biological marker, but correlation coefficients between periodontal variables and biological markers were low and great variation in biological markers was observed even at the most affected site $(\mathrm{CPI}=4)$. These findings may be partially explained by individual diversity of microbial challenge and the host response. The factor analysis summarizes many variables by using a few principal components ${ }^{10}$. Interestingly, among the biological variables, markers of the pathogenic microorganisms (PPB) showed little association with most of the markers of host response. This finding also suggests diversity of host response to the microbial challenge in periodontal tissue. Immunological variables derived from B-cells or T-cells, which were not included in the present study because of economic considerations, might provide more definite information.

We obtained 5 significant components from 14 factors in the study population. Clarification of the components can be described as follows: The first principal component, which is usually considered to be a general factor in the principal component analysis, was significantly influenced by HB, ALB, TF, AT and NE. ALB, TF and AT are factors primarily derived from serum $^{(6)}$ and $\mathrm{HB}$ is derived from gingival bleeding. Therefore, the first component may represent serum exudate and bleeding. The second component was significantly correlated with LF, MPO and LYS. Because the origin of these factors was neutrophils ${ }^{5 \text {, }}$, the component may indicate the presence of an acute inflammatory response. Neutrophil elastase is an indicator of the existence of neutrophils, but this variable was grouped with the first component rather than the second. Neutrophil elastase in GCF was studied as to enzymatic activity in relation to its inhibitors ${ }^{11}$. As the total amount of neutrophil elastase increased with the volume of GCF, NE shifted the first component. The factors LF, MPO and LYS which were extracted in the second component may offer more definite information on neutrophils than NE.
Indices of periodontal parameters such as STG, CPI-I and CPI-S were significantly correlated with the third component, indicating that the component may represent the periodontal status. Only the variables of age or PPB were extracted as significant factors in the fourth and fifth component, respectively. The fifth component may represent periodontal pathogens, because activity of PPB was determined with a unique substrate which could identify major pathogenic microorganisms $s^{3,4}$, but in the bivariate analysis, correlation of the PPB and other variables was weak, so that the variables may be considered as an independent variables among the factors in the multivariate analysis. FN was also a single variable of significance in the sixth component. Since fibronectin is one of the breakdown products of connective tissue ${ }^{5 \text {, }}$, this component may represent tissue destruction, but because variance of the component did not reach a significant level, the contribution of the component as a marker for the tissue destruction component may be weak.

The second objective of this study was to examine whether the biological markers could provide information in addition to the conventional clinical observations. In the present study, five significant principal components were extracted from 14 variables and the components were reasonably explained from the standpoint of the biological properties of the significant variables. In the principal component analysis, the factor scores for each component can be enumerated for each subject and used as an input variable in further studies ${ }^{10}$. These scores may therefore represent various aspects of the periodontal health of the site; for example, the first component as a representation of serum exudate and bleeding, and the second of acute inflammatory cells. A longitudinal study with the factor scores might be useful to monitor changes in host response to the microbial challenge among subjects in their workplace.

We selected the gingival site at the mesial approximal crevice of the first molar in the maxilla as a sampling site to monitor changes in microbial challenge and host response. This site is accessible in the limited environment of periodical oral examinations at the workplace. Furthermore, the site shows little exposure to saliva, which could disturb measurements of the crevicular sample ${ }^{(2)}$. The first molar may have a significant impact on health consciousness of workers because of the importance of the tooth for the subject. Since the early detection of periodontal disease may be of interest to workers, the effects of the sampling strategy should be considered for further investigations.

The impact of psychological stress on the immune system has been reported ${ }^{13 .}$. Psychological factors in the workplace may alter the functions of host response to periodontal microbial challenge. Recently, relationships between psychological factors and periodontal disease have been indicated ${ }^{14.15)}$. Laboratory tests involving 
various aspects of microbial challenge and host response might provide useful information on risks for progressive periodontal disease ${ }^{(6)}$. Although the variables of laboratory testing of the GCF and subgingival plaque were limited, the findings of the present pilot study suggest that further study is required to clarify the utility of laboratory testing by using 'various' biological markers in the strategy for occupational periodontal care. Establishment of the utility of laboratory testing could provide the basis for consideration of the cost-benefit of this new strategy.

\section{References}

1) Scannapieco FA. Position paper of The American Academy of Periodontology: periodontal disease as a potential risk factor for systemic diseases. J Periodontol 1998; 69: 84l-850.

2) Ainamo J, Barmes D, Beagrie G, Cutress T, Martin J, Sardo-Infirri S. Development of the World Health Organization (WHO) Community Index of Treatment Needs (CPITN). Int Dent J 1982; 32: 281-291.

3) Suido H, Eguchi T, Tanaka T, Nakamura $M$. Identification of periodontopathic bacteria based upon their peptidase activities. Adv Dent Res 1988; 2: 304 309.

4) Yoshie H, Hirose Y, Suzuki T, Hara K. Relationship between peptidase activity from Treponema denticola, Porphyromonas gingivalis, and Bacteroides forsythus and attachment loss. Periodontal Clin Investig 1996; 18: 31-38.

5) Embery G, Waddington R. Gingival crevicular fluid: biomarkers of periodontal tissuc activity. Adv Dent Res 1994; 8: 329-336.

6) Adonogianaki E, Mooney J, Kinane DF. Detection of stable and active periodontitis sites by clinical assessment and gingival crevicular acute-phase protein levels. J Periodontal Res 1996; 31: 135-143.
7) Lamster IB. In-office diagnostic tests and their role in supportive periodontal treatment. Periodontol 2000 1996; 12: 49-55.

8) Saitoh O, Matsumoto H, Sugimori K, et al. Intestinal protein loss and bleeding assessed by fecal hemoglobin, transferrin, albumin, and alpha-1-antitrypsin levels in patients with colorectal diseases. Digestion 1995; 56: $67-75$.

9) Sugi K, Saitoh O, Hirata I, Katsu K. Fecal lactoferrin as a marker for disease activity in inflammatory bowel disease: comparison with other neutrophil-derived proteins. Am Coll Gastroenterology 1996; 91: $927-$ 934.

10) SPSS Inc. Factor analysis. In: SPSS ${ }^{\circledR}$ Base 7.5 Applications Guide. Chicago: SPSS Inc; 1997: 287328.

11) Kennett CN, Cox SW, Eley BM. Localization of active and inactive elastase, alpha-1-proteinase inhibitor, and alpha-2-macroglobulin in human gingiva. J Dent Res 1995; 74: 667-674.

12) Smith QT, Freese PL. Contamination of gingival crevicular fluid (GCF) by saliva. J Dental Res 1990; 69 (Spec Iss): 326.

13) Kilcolt-Glaser JK, Kennedy S, Malkoff S, Fisher L, Speicher CE, Glaser R. Marital discord and immunity in Males. Psychosomatic Med 1988; 50: 213-229.

14) Linden GJ, Mullally BH, Freeman R. Stress and the progression of periodontal disease. J Clin Peirodontol 1996; 23: 675-680.

15) Croucher R, Marcenes WS, Torres MCMB, Hughes WS, Sheiham A. The relationship between life-events and periodontitis. A case-control study. J Clin Periodontol 1997; 24: 39-43.

16) Alpagot T, Wolff LF, Smith QT, Tran SD. Risk indicators for periodontal disease in a racially diverse urban population. J Clin Periodontol 1996; 23: 982 988. 